\title{
Resultados a largo plazo de prótesis de cúbito distal como cirugía de rescate en un caso de técnica de Sauvé-Kapandji fallida
}

\author{
V. Jiménez Díaz', D. Cecilia López', V. Guimerá García, M. Porras Moreno', \\ G. Muñoz Sánchez', F. Jara Sánchez ${ }^{1}$ \\ IServicio de Cirugía Ortopédica y Traumatología. Hospital Universitario Doce de Octubre. Madrid. España \\ 2Servicio de Cirugía Ortopédica y Traumatología. Hospital Txagorritxu de Vitoria. España
}

Resumen: La técnica de Sauvé-Kapandji se emplea como tratamiento de rescate en la patología de la articulación radiocubital distal (ARCD). Los resultados postoperatorios son buenos, aunque a largo plazo pueden surgir complicaciones como dolor, muñón cubital inestable y pérdida de fuerza de prensión. Presentamos un caso de cirugía de rescate de esta técnica mediante prótesis de cúbito distal. A los cuatro años de seguimiento, el paciente presenta desaparición completa del dolor y recuperación de la fuerza de prensión, lo que hace pensar que la prótesis cubital puede ser un buen método de rescate cuando fracasa un Sauvé-Kapandji.

Palabras clave: Muñeca, prótesis, articulación radiocubital distal.

\begin{abstract}
The Sauvé-Kapandji procedure has been widely used as a salvage method for pain and instability of distal radio ulnar joint (DRUJ). The postoperative results are good, although long term complications can occur such as painful, unstable ulnar stump and loss of grip strength. We report a case of a failed Sauvé-Kapandji procedure treated with an ulnar head prosthesis. At 4 years of follow-up, patient presents free of pain status and satisfactory grip strength recovery. Placement of an ulnar head prosthesis after a Sauvé-Kapandji procedure appears to be a reliable salvage solution.
\end{abstract}

Key words: wrist, prosthesis, distal radioulnar joint.

La degeneración de la articulación radiocubital distal (ARCD) puede dar lugar a dolor y la limitación funcional de la muñeca. El procedimiento Sauvé-Kapandji se ha usado clásicamente, en pacientes con muñecas de alta demanda para aliviar el dolor y restaurar la pronosupinación del antebrazo',2. Su ventaja teórica es que mantiene la anatomía estructural, al preservar los ligamentos ulnocarpianos, el fibrocartílago triangular y la cabeza cubital y crea un contrafuerte que proporciona un soporte mecánico que evita la traslación cubital del carpo. Sin embargo, esta técnica puede presentar como complicación, la impactación radiocubital, causada por la inestabilidad del muñón cubital y que puede alcanzar una incidencia alta ${ }^{2-5}$.

A continuación presentamos un caso clínico, con cuatro años de seguimiento, de un paciente que desarrolló una impactación radiocubital tras haber sido sometido a una técnica de Sauvé-Kapandji. Como solución para esta impactación, se propuso la colocación de una prótesis de cabeza cubital esférica, preservando la masa de fusión radiocubital, de forma similar a la técnica descrita por Fernández .

\section{CASO CLÍ́NICO}

Varón de 28 años al que se le sometió a un procedimiento de Sauvé-Kapandji como tratamiento de secuelas de una fractura de radio distal izquierdo. Tras cinco años con buena funcionalidad de la muñeca, acude a consulta por dolor y pérdida de fuerza de carácter progresivo al realizar sus actividades laborales. La exploración física demostró una impactación cubital dolorosa, además de movilidad anormal del muñón con la rotación y compresión transversa del antebrazo. El rango de movilidad era de $60^{\circ}-70^{\circ}$ para la flexo-extensión y $45^{\circ}-30^{\circ}$ para la pronosupinación. Se realizaron radio-
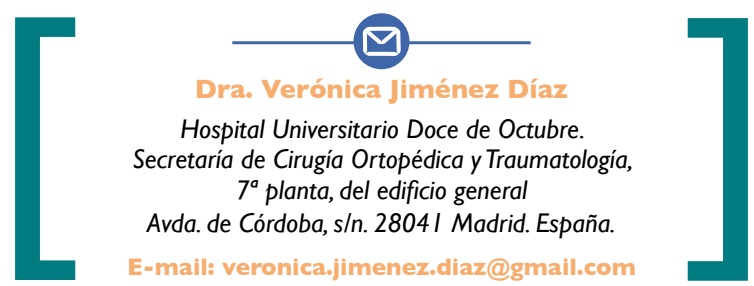


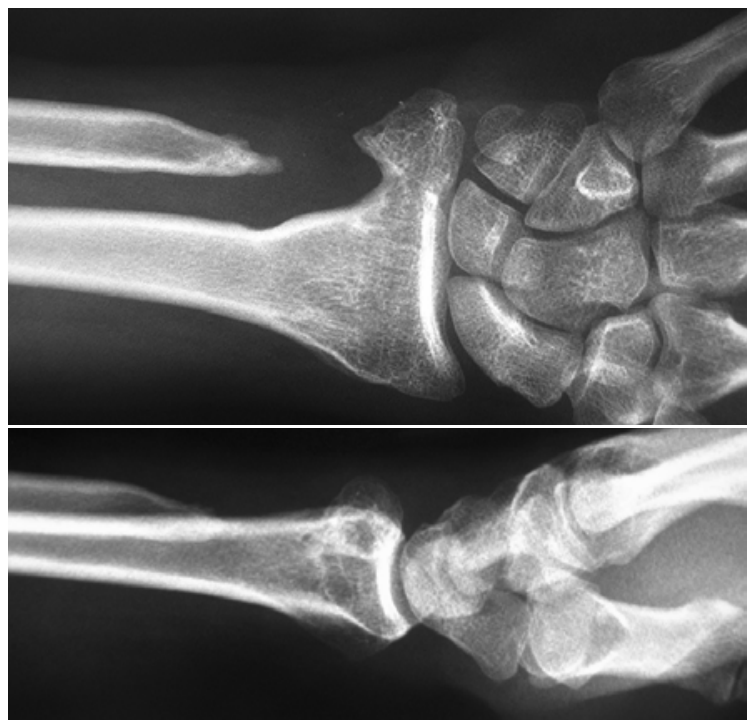

Figura I. A. y B. Radiografías de control anteroposterior y lateral, tras intervención de Sauvé-Kapandji. Se observa reabsorción del muñón cubital y signos de impactación radiocubital.

grafías de control anteroposterior y lateral, que mostraron reabsorción ósea del muñón cubital y signos de impactación radiocubital, como la muesca en la cortical medial del radio @ Figura I. Debido a estos hallazgos en las proyecciones habituales, no se realizaron radiografías de estrés con peso. Para valorar el grado de dolor se empleó la escala visual analógica (EVA), siendo su valor prequirúrgico de 3 sin carga y de 6 cuando el paciente cogía un objeto pesado.

Tras el intento fallido de tratamiento conservador mediante inmovilización y toma de antiinflamatorios no esteroideos vía oral, se decidió realizar una cirugía de rescate implantando una prótesis cubital.

La incisión se realizó sobre la cicatriz previa, llevando a cabo la disección profunda entre el quinto y sexto compartimento extensor. Se realizó la apertura con punzón del borde distal del muñón cubital para llevar a cabo una resección reglada de un centímetro mediante una sierra oscilante. A continuación, se prepararon la fosita para la cabeza cubital con fresas esféricas y el canal para alojar el vástago de la prótesis. Se implantaron los componentes de prueba para comprobar la estabilidad del implante y posteriormente, el vástago, el cuello y la cabeza definitivos no cementados (prótesis cubital modular Ascension First Choice Modular Ulnar Head con cabeza de cromo-cobalto y vástago de titanio), realizando su reducción mediante tracción longitudinal.

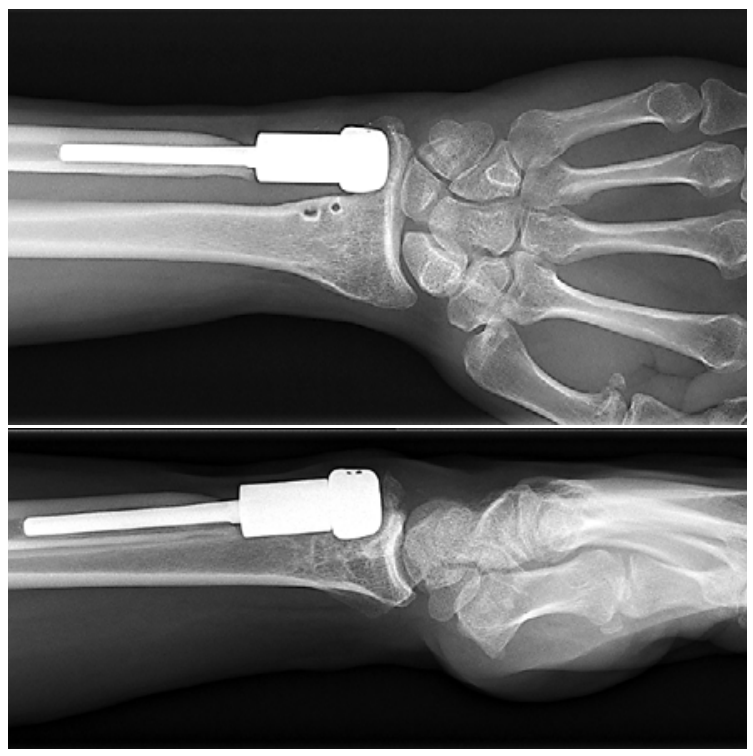

Figura 2. A. y B. Radiografías de control anteroposterior y lateral a los cuatro años de la colocación de la prótesis cubital.

Antes de llevar a cabo el cierre definitivo se realizó una plastia con tendón del palmaris longus alrededor del cuello de la prótesis, que se suturó junto a estructuras capsulo-retinaculares para dar mayor estabilidad al implante.

Tras la inmovilización inicial con una férula antebraquial, a las dos semanas el paciente comenzó con ejercicios de pronosupinación y a las seis, retomó sus actividades habituales con remisión completa de la sintomatología.

A los cuatro años de seguimiento, la prótesis es estable y la movilidad ha mejorado, presentando un rango actual de $80^{\circ}-80^{\circ}$ para la flexo-extensión y pronosupinación prácticamente completa. El dolor ha desparecido, siendo la puntuación en la escala EVA de 0 con y sin carga de peso. La fuerza de prensión ha aumentado de $5 \mathrm{~kg}$ en el momento previo a la cirugía a $18 \mathrm{~kg}$ tras la intervención, lo que supone un $64 \%$ del miembro contralateral. Las radiografías de control no muestras signos de aflojamiento ni signos de reabsorción del cuello por anclaje diafisario (aigura 2.

\section{DISCUSIÓN}

Las alteraciones de la ARCD se manifiestan por dolor, inestabilidad y pérdida de fuerza. Agotadas las opciones de tratamiento conservador, en pacientes con alta demanda funcional, se han descrito técnicas quirúrgicas 
de resección cubital distal como los procedimientos de Darrach o Bowers.

El método de Sauvé-Kapandji se describe por primera vez en 1936 como rescate para esta patología mediante la creación de una masa de artrodesis RCD asociada a una resección cubital distal',2. Múltiples series como las de Gordon, Millroy o Sanders muestran resultados variables con mejoría significativa del dolor y balance articular, ${ }^{3,5}$. La complicación más frecuentemente descrita, es un síndrome de impactación del muñón cubital que provoca un pinzamiento entre el muñón y el radio, manifestándose a través de chasquidos y dolor. Puede ser demostrado radiográficamente mediante proyecciones anteroposteriores de estrés, en las que el paciente, con el puño cerrado y el codo en flexión de $90^{\circ}$ soporta una carga ligera de $1-2 \mathrm{~kg}$; observándose en la imagen la convergencia entre el muñón cubital y la diáfisis radial, que en algunos casos puede presentar una muesca o escalón en la cortical medial ${ }^{6}$.

Los procedimientos de rescate en estos pacientes incluyen, la resección proximal del muñón, la sinóstosis radiocubital y la estabilización del muñón cubital mediante tenodesis del extensor carpi ulnaris (ECU) o del flexor carpi ulnaris (FCU); que a pesar de haber demostrado buenos resultados iniciales respecto a disminución de dolor y mejoría de la estabilidad del muñón, pueden acabar deteriorándose con el tiempo ${ }^{7-10}$.

Como alternativa a estos métodos surgieron las prótesis cubitales. Desde el primer modelo descrito por Swanson" hasta nuestros días, se han desarrollado diversos tipos de prótesis que incluyen desde hemiartroplastias como la prótesis de Herbert ${ }^{12}$, hasta artroplastias totales de la ARCD como la prótesis de Sche$k^{2} r^{13,14}$. Múltiples estudios han demostrado excelentes resultados clínico-radiológicos con escaso número de complicaciones, aunque muchos de ellos presentan un corto periodo de seguimiento, 12-15.

En el presente caso, se implantó una prótesis cubital siguiendo la técnica quirúrgica publicada por Fernández ${ }^{6}$ en 2006, que da estabilidad a la prótesis creando una fosita en la masa de artrodesis radiocubital, siendo así innecesario el tratamiento de las partes blandas, que puede ser dificultoso en pacientes que ya han sido sometidos a intervenciones quirúrgicas previas. Se descartó la artroplastia total de entrada, por ser una técnica más agresiva y que además podía constituir una opción futura de rescate, en el caso de que los resultados de la hemiartroplastia no fueran satisfactorios. La única diferencia con el método original, es que no se realizó una osteotomía radial distal, para evitar aumentar la morbilidad del procedimiento, este es el motivo por el que las radiografías de control muestran una subluxación de la prótesis, que clínicamente es asintomática.

A pesar de esta diferencia en la técnica, el resultado clínico es similar a los obtenidos por Fernández en su serie, logrando estabilidad postquirúrgica a nivel cubital distal, desaparición del dolor, mejoría de la movilidad y de la fuerza de prensión. Respecto a la subluxación dorsal de la prótesis, desconocemos qué efectos puede ocasionar a largo plazo, pudiendo permanecer asintomática o ser fuente de dolor o limitación de movilidad de muñeca y antebrazo.

Recientemente, van Shoonhoven y cols ${ }^{16}$ han revisado su antigua serie de 23 pacientes con prótesis cubital, obteniendo excelentes resultados en un seguimiento a largo plazo (once años). Por la experiencia con el presente caso y de acuerdo con la bibliografía, pensamos que la prótesis de cúbito puede ser una alternativa de rescate para una impactación radiocarpiana tras una cirugía de Sauvé-Kapandji.

\section{CONFLICTOS DE INTERESES}

Los autores declaran no tener conflictos de intereses.

\section{BIBLIOGRAFÍA}

I. Sauvé L, Kapandji M. Nouvelle technique de traitement chirurgical des luxations récidivantes isolées de l'extrémité inferieure du cubitus. J Chirurg (Paris). 1936:47:589-594.

2. Slater R. The Sauvé-Kapandji procedure. J Hand Surg Am. 2008; 33A: $1632-1638$.

3. Gordon L, Levinsohn DG, Moore SV, Dodds RJ, Castelman LD. The Sauvé-Kapandji procedure for the treatment of posttraumatic distal radioulnar joint problems. Hand Clin. 1991;7:397-403.

4. Millroy P, Coleman S. Ivers R. The Sauvé-Kapandji operation: Technique and results. J Hand Surg Br. |992; |7:4||-4|4.

5. Sanders RA, Frederick HA, Hontas RB. The SauvéKapandji procedure: a salvage operation for the distal radioulnar joint. J Hand Surg Am. 199 |; | 6: I | 251129.

6. López Fernández D, Joneschild E, Abella D. Treatment of Failed Sauvé-Kapandji Procedures with a 
Spherical Ulnar Head Prosthesis. Clin Orthop Rel Res. 2006;445: I00- 107.

7. Minami A, Kato H, Iwasaki N. Modification of the Sauvé-Kapandji procedure with extensor carpi ulnaris tenodesis. J Hand Surg Am. 2000;25: I0801084

8. Couturier C, Alnot JY, Masmejean E. Instabilité dorsale du moignon ulnaire après résection diaphysaire distale: stabilisation avec un hémi extensor carpi ulnaris. Chir Main. 2002;21:242-251.

9. Lamey DM, Fernández DL. Results of the modified Sauvé-Kapandji procedure in the treatment of chronic posttraumatic derangement of the distal radioulnar joint. J Bone Joint Surg Am. 1998;80A: 1758-1769.

10. Laurentin LA, Goodwin AN, Babb BA, Scheker LR. A study of functional outcomes following implantation of a total distal radioulnar joint prosthesis. J Hand Surg EurVol 2008;33: I 8-28.

I I. Swanson AB. Implant arthroplasty for disabilities of the distal radioulnar joint. Use of a silicone rubber capping implant following resection of the ulnar head. Orthop Clin North Am. 1973;4:373-382.

12. Herbert TJ, van Schoonhoven J. Ulnar head prostheses: a new solution for problems at the distal radioulnar joint. en: Simmer BR, Allieu Y, Lluch A, Stanley J, eds. Hand arthroplasties. London: Martin Dunitz; 2000. p. I45-| 49.

13. Scheker LR, Babb BA, Killion PE. Distal ulnar prosthetic replacement. Orhop Clin North Am. 200।:32:365-376.

14. Scheker LR. Implant arthroplasty for the distal radioulnar joint. J Hand Surg Am 2008;33A: I 639- 1644.

I5. van Schoonhoven J, Fernandez DL, Bowers WH, HerbertTJ. Salvage of failed resection arthroplasties of the distal radioulnar joint using a new ulnar head prosthesis. J Hand Surg Am. 2000;25(3):438-446.

16. van Schoonhoven J, Fernandez DL, Bowers WH, Herbert TJ. Salvage of failed resection arthroplasties of the distal radioulnar joint using an ulnar head prosthesis: long-term results. I Hand Surg Am. 20 I2 Jul;37(7): | 372-80. 